\title{
ASYMPTOTIC BEHAVIOR OF SOLUTIONS OF VOLTERRA INTEGRO-DIFFERENTIAL EQUATIONS
}

\author{
M. RAMA MOHANA RAO AND P. SRINIVAS
}

\begin{abstract}
The asymptotic behavior of solutions of Volterra integrodifferential equations of the form

$$
x^{\prime}(t)=A(t) x(t)+\int_{0}^{t} K(t, s) x(s) d s+F(t)
$$

is discussed in which $A$ is not necessarily a stable matrix. An equivalent equation which involves an arbitrary function is derived and a proper choice of this function would pave a way for the new coefficient matrix $B$ (corresponding $A)$ to be stable.
\end{abstract}

1. Introduction. The objective of this paper is to investigate the asymptotic behavior of solutions of the Volterra integro-differential equation (VIDE)

$$
x^{\prime}(t)=A(t) x(t)+\int_{0}^{t} K(t, s) x(s) d s+F(t)
$$

where $A(t)$ and $K(t, s)$ are $n \times n$ matrices defined and continuous on $0 \leq t<\infty$ and $0 \leq s \leq t<\infty$, respectively, and $x(t)$ and $F(t)$ are $n$-vectors with $F(t)$ continuous on $0 \leq t<\infty$, when the matrix $A$ is not necessarily stable. Our main approach here is by way of deriving an equivalence theorem (Lemma 2.1) which has the potential to supply us a stable matrix $B$ corresponding to $A$.

It is well known that the linear autonomous ordinary differential system is asymptotically stable if all the characteristic roots of the coefficient matrix have negative real parts [12, Chapter 3]. For nonautonomous systems, with an addition of the Lipschitz condition on the coefficient matrix, similar results have been expounded in [4 and 5]. Thus while studying VIDE (1.1), be it through Liapunov second method $[1,2,14,15]$ or from perturbation theory $[8,10,13]$, it has invariably been assumed that the coefficient matrix is stable. Notable exceptions that have dispensed with the stability condition on the coefficient matrix have been the works of Levin [9], Grossman and Miller [7], Grimmer and Seifert [6], Burton [3], among others. In [9] this has been done by defining a suitable energy function while in [7] the integrability of the resolvent function of VIDE (1.1) has been characterized by a transformation condition similar to that given in [11] for Volterra integral equations. In [6] the same has been achieved by studying the rsolvent of a transformed equation. Quite recently in [3], the conditions involving the anti-derivatives of the kernel are assumed. Motivated by the interesting nature of this problem, an attempt has been made in $\S 2$ to study the asymptotic behavior of solutions of $(1.1)$

Received by the editors October 3, 1983 and, in revised form, March 26, 1984.

1980 Mathematics Subject Classification. Primary 45D05; Secondary 34K20.

Key words and phrases. Integro-differential equation, equivalent equation, arbitrary function, differential resolvent. 
when the coefficient matrix $A$ in (1.1) is not necessarily stable. Our approach here has been to develop an equivalent equation which involves an arbitrary function. A proper choice of this function would pave a way for the new coefficient matrix (corresponding to equivalent VIDE) to be stable.

2. Main results. The following lemmas are useful in our subsequent discussion.

LEMMA 2.1. Let $\Phi(t, s)$ be an $n \times n$ continuously differentiable matrix function on $0 \leq s \leq t<\infty$. Then the equation (1.1) is equivalent to

$$
y^{\prime}(t)=B(t) y(t)+\int_{0}^{t} L(t, s) y(s) d s+H(t), \quad y(0)=x_{0},
$$

where

$$
\begin{aligned}
B(t) & =A(t)-\Phi(t, t) \\
L(t, s) & =K(t, s)+\Phi_{s}(t, s)+\Phi(t, s) A(s)+\int_{s}^{t} \Phi(t, u) K(u, s) d u
\end{aligned}
$$

and

$$
H(t)=F(t)+\Phi(t, 0) x_{0}+\int_{0}^{t} \Phi(t, s) F(s) d s .
$$

PROOF. Let $x(t)$ with $x(0)=x_{0}$ be any solution of (1.1) existing on the interval $0 \leq t<\infty$. Consider the identity

$$
\int_{0}^{t} \Phi_{s}(t, s) x(s) d s=\Phi(t, t) x(t)-\Phi(t, 0) x_{0}-\int_{0}^{t} \Phi(t, s) x^{\prime}(s) d s .
$$

Substituting for $x^{\prime}(t)$ from (1.1) and using Fubini's theorem, we get

$$
\begin{aligned}
\int_{0}^{t} \Phi_{s}(t, s) & x(s) d s=\Phi(t, t) x(t)-\Phi(t, 0) x_{0}-\int_{0}^{t} \Phi(t, s) F(s) d s \\
& -\int_{0}^{t} \Phi(t, s) A(s) x(s) d s-\int_{0}^{t}\left[\int_{\tau}^{t} \Phi(t, s) K(s, \tau) d s\right] x(\tau) d \tau .
\end{aligned}
$$

Then it follows from (1.1) and (2.1)-(2.3) that

$$
\begin{aligned}
\int_{0}^{t} L(t, s) x(s) d s= & \int_{0}^{t} K(t, s) x(s) d s+\int_{0}^{t} \Phi_{s}(t, s) x(s) d s \\
& +\int_{0}^{t} \Phi(t, s) A(s) x(s) d s \\
& +\int_{0}^{t}\left[\int_{s}^{t} \Phi(t, u) K(u, s) d u\right] x(s) d s \\
= & x^{\prime}(t)-A(t) x(t)-F(t)+\Phi(t, t) x(t) \\
& -\Phi(t, 0) x_{0}-\int_{0}^{t} \Phi(t, s) F(s) d s \\
= & x^{\prime}(t)-B(t) x(t)+H(t) .
\end{aligned}
$$

Thus every solution of (1.1) is also a solution of (2.1). Conversely, let $y(t)$ be a solution of $(2.1)$ with $y(0)=x_{0}$. Define

$$
z(t)=y^{\prime}(t)-F(t)-A(t) y(t)-\int_{0}^{t} K(t, s) y(s) d s .
$$


From (2.1), (2.2) and the definition of $z(t)$ we obtain

$$
\begin{aligned}
z(t)= & -\Phi(t, t) y(t)+\int_{0}^{t}\left[\Phi_{s}(t, s)+\Phi(t, s) A(s)+\int_{s}^{t} \Phi(t, u) K(u, s) d u\right] y(s) d s \\
& +\Phi(t, 0) x_{0}+\int_{0}^{t} \Phi(t, s) F(s) d s .
\end{aligned}
$$

Substituting for $F(s)$ from the definition of $z(t)$ and changing the order of integration, we get

$$
\begin{aligned}
z(t)= & -\left[\Phi(t, t) y(t)-\Phi(t, 0) x_{0}-\int_{0}^{t} \Phi(t, s) y^{\prime}(s) d s\right] \\
& +\int_{0}^{t} \Phi_{s}(t, s) y(s) d s-\int_{0}^{t} \Phi(t, s) z(s) d s
\end{aligned}
$$

From the identity

$$
\int_{0}^{t} \Phi(t, s) y^{\prime}(s) d s=\Phi(t, t) y(t)-\Phi(t, 0) x_{0}-\int_{0}^{t} \Phi_{s}(t, s) y(s) d s
$$

it is clear that

$$
z(t)=-\int_{0}^{t} \Phi(t, s) z(s) d s .
$$

Since $\Phi(t, s)$ is continuous, it follows from the uniqueness of solutions of Volterra integral equations that $z(t)=0$. Hence $y(t)$ solves (1.1).

REMARK 2.2. It is to be noted that if $\Phi(t, s)$ is the differentiable resolvent corresponding to the kernel $K(t, s)$, then the equation (2.1) together with (2.2) gives the usual variation of constants formula (see Grossman and Miller [8]).

LEMMA 2.3. Let $B(t)$ be an $n \times n$ continuous matrix which commutes with its integral and let $M$ and $\alpha$ be positive numbers. Suppose the inequality

$$
\left|\exp \left(\int_{s}^{t} B(\tau) d \tau\right)\right| \leq M e^{-\alpha(t-s)}, \quad 0 \leq s \leq t<\infty,
$$

holds. Then every solution $x(t)$ of (2.1) with $x(0)=x_{0}$ satisfies

$$
\begin{aligned}
|x(t)| \leq & M\left|x_{0}\right| e^{-\alpha t}+M \int_{0}^{t} e^{-\alpha(t-\tau)}|H(\tau)| d \tau \\
& +M \int_{0}^{t}\left[\int_{s}^{t} e^{-\alpha(t-\tau)}|L(\tau, s)| d \tau\right]|x(s)| d s .
\end{aligned}
$$

PROOF. Multiplying both sides of $(2.1)$ by $\exp \left(-\int_{0}^{t} B(\tau) d \tau\right)$ and rearranging the terms, we obtain

$$
\left(\exp \left(-\int_{0}^{t} B(\tau) d \tau\right) x(t)\right)^{\prime}=\exp \left(-\int_{0}^{t} B(\tau) d \tau\right)\left[H(t)+\int_{0}^{t} L(t, s) x(s) d s\right] .
$$

Integrating from 0 to $t$, we get

$$
\begin{aligned}
\exp \left(-\int_{0}^{t} B(\tau) d \tau\right) x(t)= & x_{0}+\int_{0}^{t} \exp \left(-\int_{0}^{s} B(\tau) d \tau\right) H(s) d s \\
& +\int_{0}^{t} \exp \left(-\int_{0}^{s} B(\tau) d \tau\right)\left(\int_{0}^{s} L(s, u) x(u) d u\right) d s
\end{aligned}
$$


By changing the order of integration on the right side and using (2.4), we obtain (2.5).

REMARK 2.4. If $B$ is a constant matrix, then it commutes with its integral. Further, the condition (2.4) holds if, in addition, all the characteristic roots of $B$ have negative real parts (cf. [12, Chapter 2]).

THEOREM 2.5. Let $\Phi(t, s)$ be a continuously differentiable $n \times n$ matrix function such that, for $0 \leq s \leq t<\infty$,

(i) the hypotheses of Lemma 2.3 holds,

(ii) $|\Phi(t, s)| \leq L_{0} e^{-\gamma(t-s)}$,

(iii) $\sup _{0 \leq s \leq t<\infty} \int_{s}^{t} e^{\alpha(\tau-s)}|L(\tau, s)| d \tau \leq \alpha_{0}$,

where $L_{0}, \gamma(>\alpha), \alpha_{0}$ are positive real numbers. Suppose further

(iv) $F(t) \equiv 0$,

where $F(t)$ is defined in (1.1). If $\alpha-M \alpha_{0}>0$, then every solution $x(t)$ of (1.1) tends to zero exponentially as $t \rightarrow+\infty$.

PROOF. In view of Lemma 2.1 and the function $\Phi(t, s)$ satisfying the conditions (i), (ii) and (iii), it is enough to show that every solution of (2.1) tends to zero exponentially as $t \rightarrow+\infty$. Since $F(t) \equiv 0$, the equation (2.2) and (2.5) and the condition (ii) imply that

$$
\begin{aligned}
e^{\alpha t}|x(t)| \leq & M\left|x_{0}\right|+M L_{0}\left|x_{0}\right| \int_{0}^{t} e^{-(\gamma-\alpha) \tau} d \tau \\
& +M \int_{0}^{t}\left[\int_{s}^{t} e^{\alpha \tau}|L(\tau, s)| d \tau\right]|x(s)| d s
\end{aligned}
$$

Using (iii), we get

$$
e^{\alpha t}|x(t)| \leq M\left|x_{0}\right|+\frac{M L_{0}\left|x_{0}\right|}{(\gamma-\alpha)}+\int_{0}^{t} M \alpha_{0} e^{\alpha s}|x(s)| d s .
$$

The application of Gronwall inequality yields that

This implies that

$$
e^{\alpha t}|x(t)| \leq M\left|x_{0}\right|\left(1+\frac{L_{0}}{(\gamma-\alpha)}\right) e^{M \alpha_{0} t} .
$$

$$
|x(t)| \leq M\left|x_{0}\right|\left(1+\frac{L_{0}}{(\gamma-\alpha)}\right) e^{-\left(\alpha-M \alpha_{0}\right) t} .
$$

Thus in view of $\alpha-M \alpha_{0}>0$, the result follows.

COROLLARY 2.6. In addition to the assumptions (i), (ii) and (iv) of Theorem 2.5, suppose the following conditions hold:

(a) $|K(t, s)| \leq K_{0} e^{-\beta(t-s)}$ for $0 \leq s \leq t<\infty$,

(b) $\left|\Phi_{s}(t, s)\right| \leq N_{0} e^{-\delta(t-s)}$ for $0 \leq s \leq t<\infty$,

(c) $|A(t)| \leq A_{0}$ for $0 \leq t<\infty$

where $A_{0}, N_{0}, K_{0}, \beta, \delta$ are positive real numbers, and

(d) $\gamma>\beta>\alpha, \delta>\alpha$ and $\alpha-M \hat{\alpha}_{0}>0$ where

$$
\hat{\alpha}_{0} \stackrel{\text { def }}{=}\left[\frac{K_{0}}{\beta-\alpha}+\frac{N_{0}}{\delta-\alpha}+\frac{L_{0} A_{0}}{\gamma-\alpha}+\frac{K_{0} L_{0}}{(\beta-\alpha)(\gamma-\beta)}\right] .
$$

Then every solution $x(t)$ of (1.1) tends to zero exponentially as $t \rightarrow+\infty$. 
ProOF. Following the proof of Theorem 2.5, we obtain

$$
\begin{aligned}
e^{\alpha t}|x(t)| \leq & M\left|x_{0}\right|\left(1+\frac{L_{0}}{(\gamma-\alpha)}\right) \\
& +M \int_{0}^{t}\left[\int_{s}^{t} e^{\alpha \tau} \mid K(\tau, s)+\Phi_{s}(\tau, s)+\Phi(\tau, s) A(s)\right. \\
& \left.+\int_{s}^{\tau} \Phi(\tau, u) K(u, s) d u \mid d \tau\right]|x(s)| d s
\end{aligned}
$$

Using conditions (i), (a), (b), (c) and estimating each integral on the right side of (2.6), we get

$$
\begin{aligned}
e^{\alpha t}|x(t)| \leq & M\left|x_{0}\right|\left(1+\frac{L_{0}}{(\gamma-\alpha)}\right) \\
& +\int_{0}^{t} M \hat{\alpha}_{0} e^{\alpha s}|x(s)| d s .
\end{aligned}
$$

Thus, in view of condition (d), the application of Gronwall's inequality yields the desired result.

REMARK 2.7. If $F(t) \equiv 0$ in equation (1.1), then the Theorem 2.5 asserts that the zero solution of $(1.1)$ is exponentially asymptotically stable.

REMARK 2.8. If $F(t)$ is not zero in Theorem 2.5, still the solutions of (1.1) tends to zero as $t \rightarrow+\infty$ provided $\int_{0}^{\infty}|F(s)| d s<\infty$. This is an immediate consequences of variation of constants formula (see $[8]$ ) and Theorem 2.5 .

REMARK 2.9. It is possible to select a matrix function $\Phi(t, s)$ satisfying the conditions (i) and (ii) of Theorem 2.5 and condition (b) of Corollary 2.6. For example, if $\Phi(t, s)=L_{0} e^{-\gamma(t-s)} I$, then $N_{0}=L_{0} \gamma$ and $\delta=\gamma . \Phi(t, t)$ being a constant matrix in this case, the estimate (2.4) is guaranteed if $A(t)$ is a constant matrix and $B$ is negative definite.

REMARK 2.10. Basically it is the condition " $M \hat{\alpha}_{0}<\alpha$ " in Corollary 2.6 which controls the asymptotic nature of the solution $x(t)$ of (1.1). A look at the composition of $\hat{\alpha}_{0}$ reveals that while so choosing $\gamma$ and $\delta$ much away from $\beta$ and $\alpha$, respectively, we can nullify the effect of the last three terms in $\hat{\alpha}_{0}$, the first term $K_{0} /(\beta-\alpha)$ being the essential term which we have to reckon with. Therefore, if $\beta$ is so large as to exceed $\left(\alpha^{2}+M K_{0}\right) / \alpha$, then $M \hat{\alpha}_{0}$ would be less than $\alpha$. Thus we see that the attenuation required on the kernel $K(t, s)$ is linked with the constant $\alpha$ in (2.4). This conclusion implicity assumed the estimate (2.4). Such an estimate would be possible when the transformed matrix $B$ is constant and negative definite.

REMARK 2.11. In [3], a condition of the type (2.4) has been used for the matrix $Q \stackrel{\text { def }}{=}(A(t)-G(t, t))$, where $G(t, s)$ is the anti-derivative of the kernel $K(t, s)$ (i.e. $\partial G(t, s) / \partial t=K(t, s))$. As such the matrix $B$ in our study allows more flexibility due to the arbitrary character of the function $\Phi(t, s)$. Further, our approach is entirely different and the analysis in [3] can be applied to equation (2.1) in order to obtain sharper estimates. Thus our Theorem 2.5 is in addition to the Theorem 2 of $[3]$ rather than a substitute for it.

EXAMPLE 2.12. In (1.1) (scalar case), let $A(t)=a_{1} e^{-b_{1} t}-a_{2}, K(t, s)=$ $e^{-b_{2}(t, s)}$ and $F(t) \equiv 0$ where $a_{1}, a_{2}, b_{1}, b_{2}$ are positive real numbers. Choose 
$\Phi(t, s)=a_{1} e^{-b_{1} t}$. Then $M=1, \alpha=a_{2}, A_{0}=a_{1}+a_{2}, L_{0}=a_{1}, K_{0}=1, \beta=$ $b_{2}, \gamma=\delta=b_{1}$ and $N_{0}=0$. Thus the condition (d) of Corollary 2.6 holds if $a_{1}=\hat{K} a_{2}, b_{1}=\left(4 \hat{K}^{2}+4 \hat{K}+1\right) a_{2}, b_{2}=\left(4+a_{2}^{2}\right) / a_{2}$ and $a_{2} \geq 2 /[\hat{K}(4 \hat{K}+3)]^{1 / 2}$ where $\hat{K}(1<\hat{K}<\infty)$ is an arbitrary real number. For example, if $\hat{K}=2, a_{2}=1$, then $M \hat{\alpha}_{0} \simeq 0.53$ and $\alpha=1$.

The authors wish to thank the referee for suggesting a proof for the converse part of Lemma 2.1.

\section{REFERENCES}

1. T. A. Burton, Stability theory for Volterra equations, J. Differential Equations 32 (1979), 101-118.

2. __ Uniform stability for Volterra equations, J. Differential Equations 36 (1980), 40-53.

3. __ An integro-differential equation, Proc. Amer. Math. Soc. 79 (1980), 393-399.

4. W. A. Coppel, Stability and asymptotic behavior of differential equations, Heath, Boston, Mass., 1965.

5. __ Dichotomies in stabrility theory, Lecture Notes in Math., vol. 629, Springer-Verlag, Berlin and New York, 1978.

6. R. Grimmer and G. Seifert, Stability properties of Volterra integro-differential equations, J. Differential Equations 19 (1975), 142-166.

7. S. I. Grossman and R. K. Miller, Nonlinear Volterra integro-differential systems with $L^{1}$-kernels, J. Differential Equations 13 (1973), 551-566.

8. __ Perturbation theory for Volterra integra-differential systems, J. Differential Equations 8 (1970), 451-474.

9. J. J. Levin, The asymptotic behavior of the solution of a Volterra equation, Proc. Amer. Math. Soc. 14 (1963), 534-541.

10. V. Lakshmikantham and M. Rama Mohana Rao, Integro-differential equations and extension of Liapunov's method, J. Math. Anal. Appl. 30 (1970), 435-447.

11. R. E. A. C. Paley and N. Wiener, Fourier transforms in complex domain, Amer. Math. Soc., Providence, R.I., 1934.

12. M. Rama Mohana Rao, Ordinary differential equations-Theory and applications, Edward Arnold, London, 1981.

13. M. Rama Mohana Rao and Chris P. Tsokos, Integro-differential equations of Volterra type, Bull. Austral. Math. Soc. 3 (1970), 9-22.

14. G. Seifert, Liapunov-Razumilchin conditions for stability and boundedness of functional differential equations of Volterra type, J. Differential Equations 14 (1973), 424-430.

15. L Liapunov-Razumikhin conditions for asymptotic stability in functional differential equations of Volterra type, J. Differential Equations 16 (1974), 289-298.

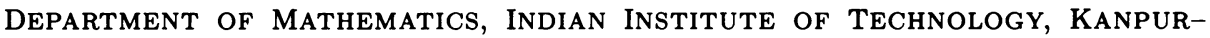
208016, INDIA

Current address: Department of Computer Science, University of Houston, University Park, Houston, Texas 77004 